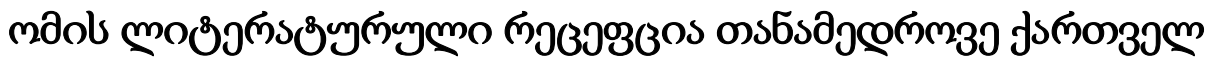

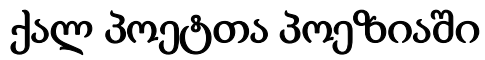

\author{
zмzosపुomo бoбm \\ os 3 m 8 zмдj

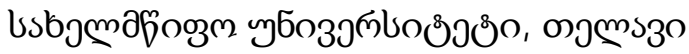 \\ https://doi.org/10.52340/idw.2021.519
}

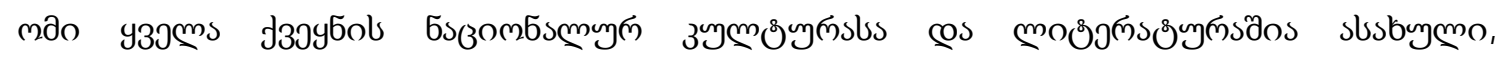

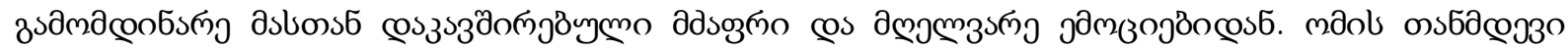

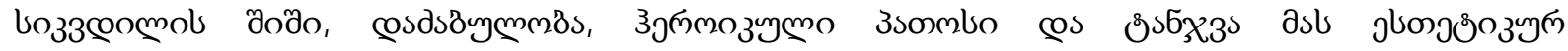

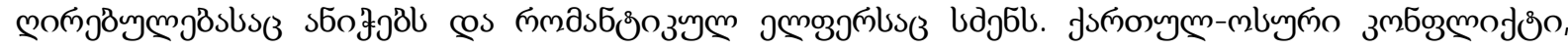

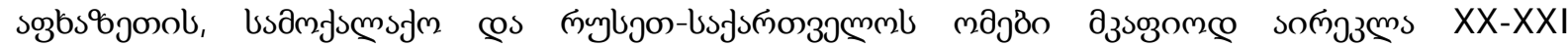

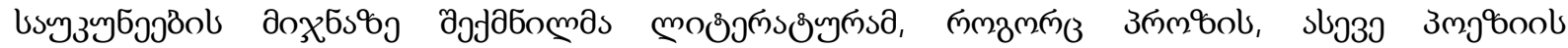

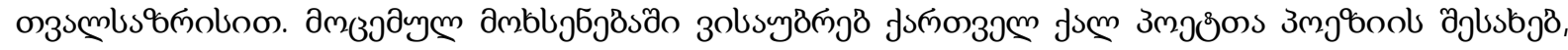

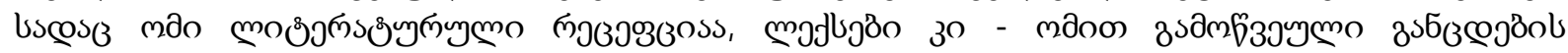

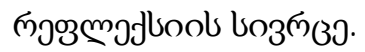

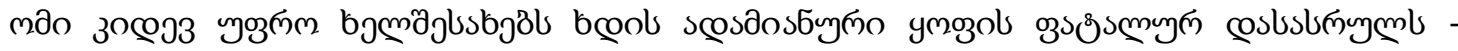

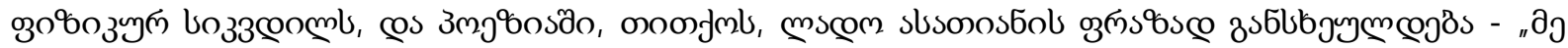

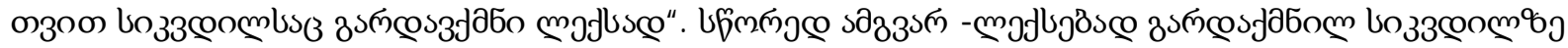

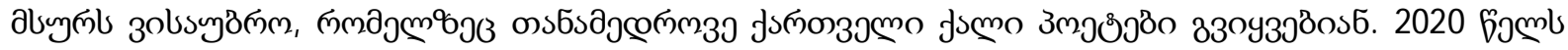

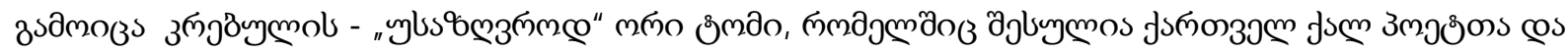

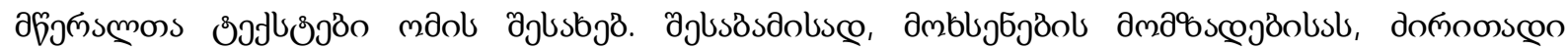

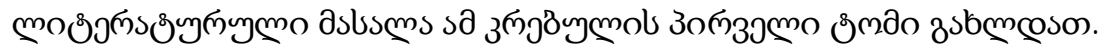

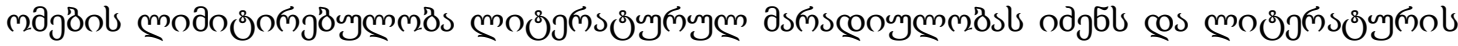

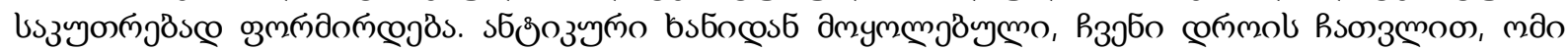

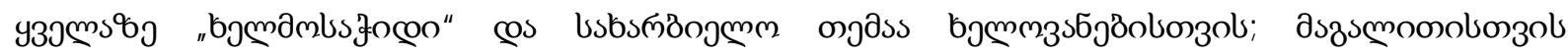

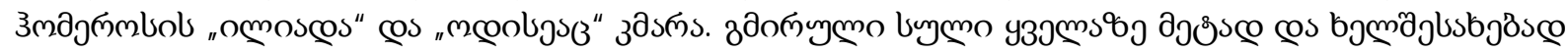

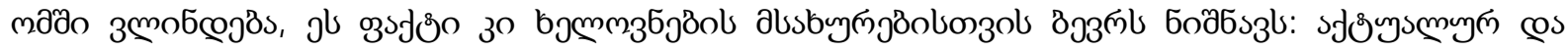

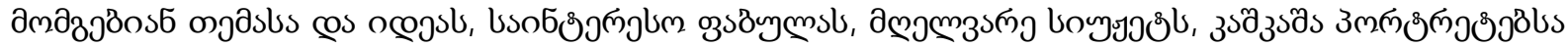

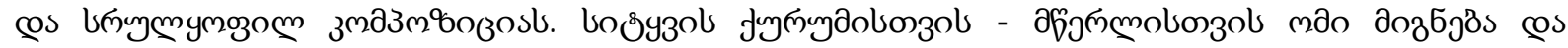

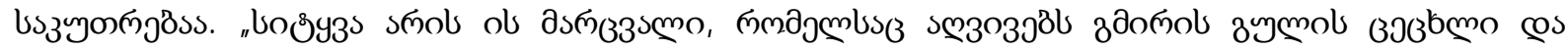

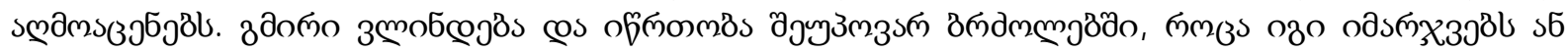

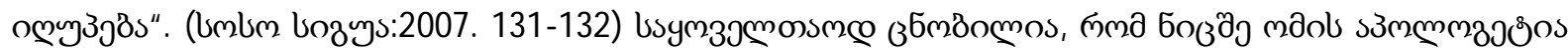

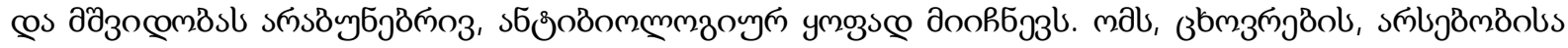

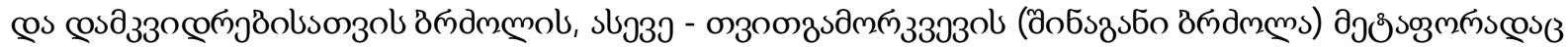

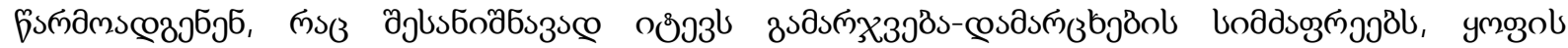

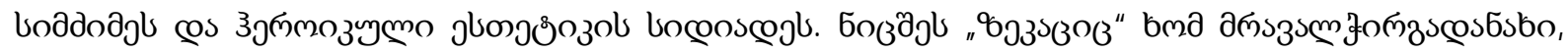

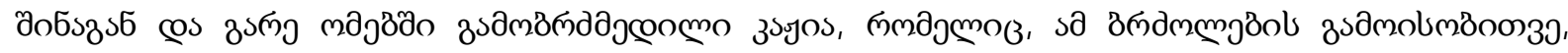

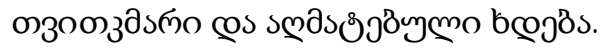

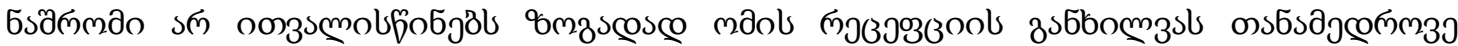

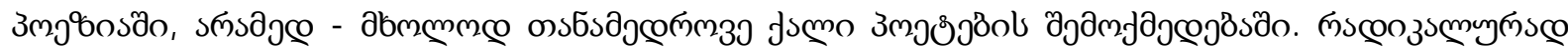

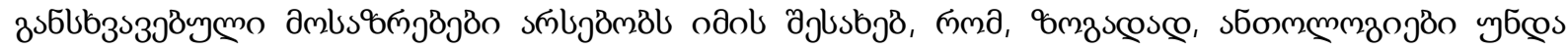

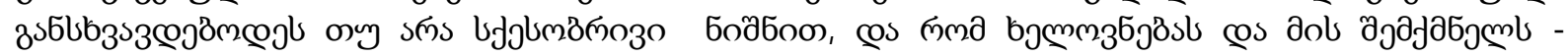




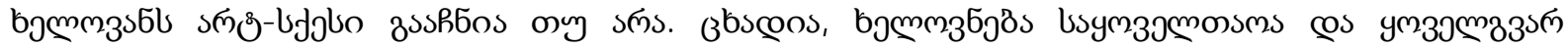

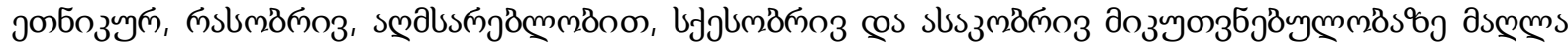

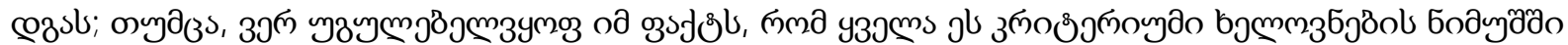

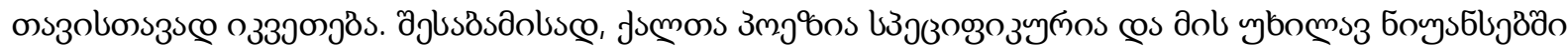

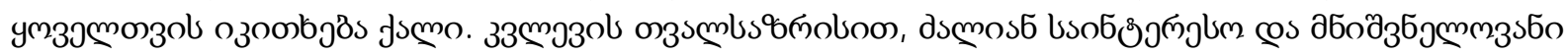

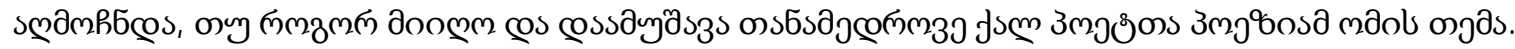

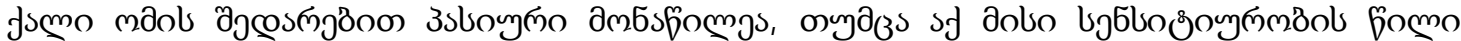

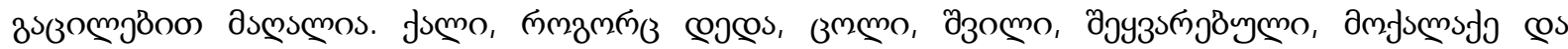

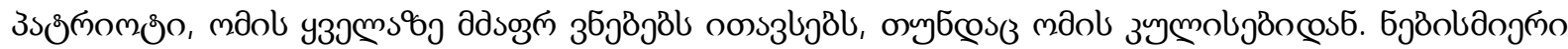

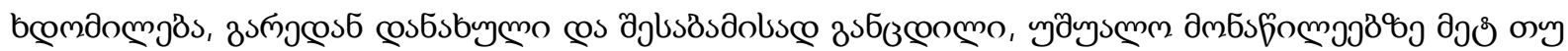

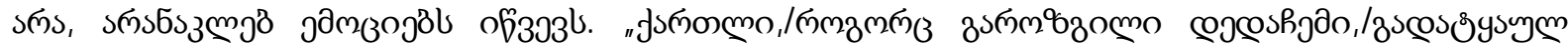

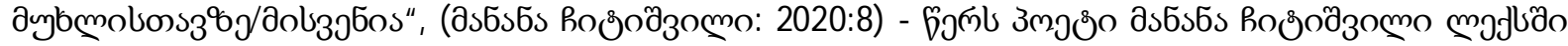

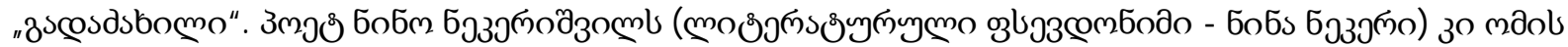

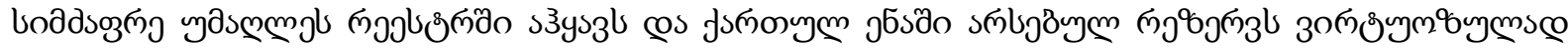
оyjбjßl ylssonsynm mojkд̃o:

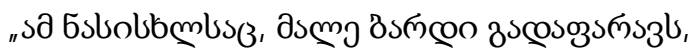

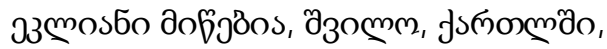

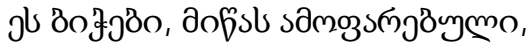

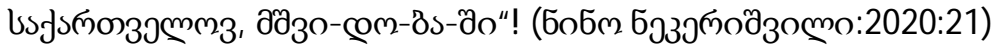

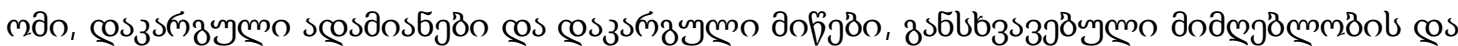

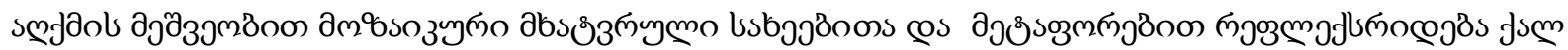

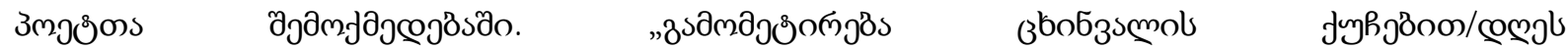

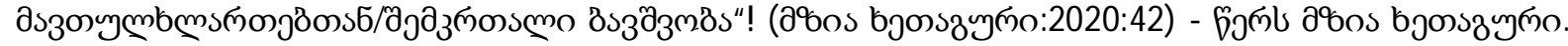

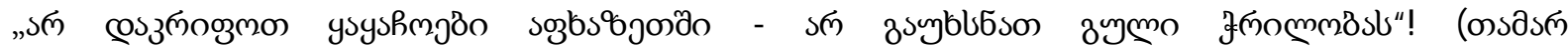

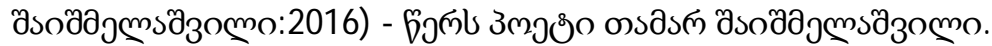

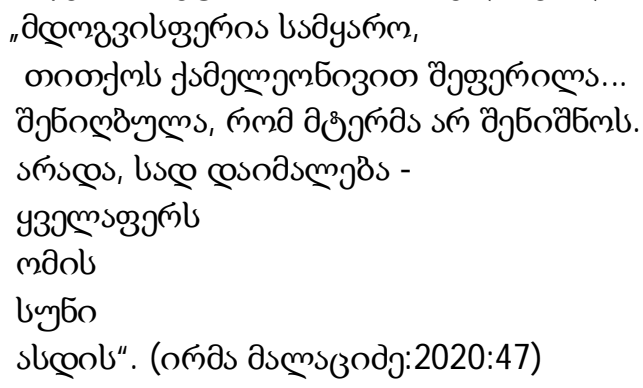

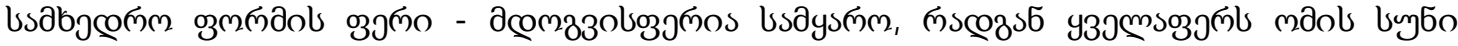

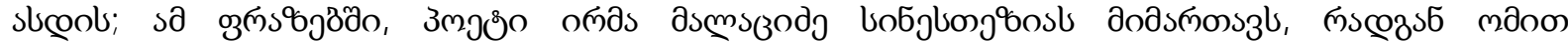

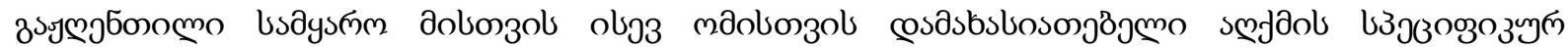

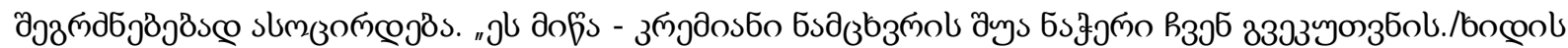

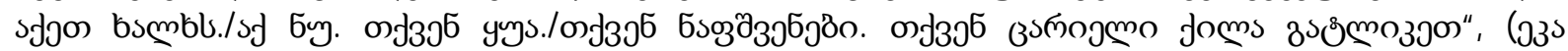

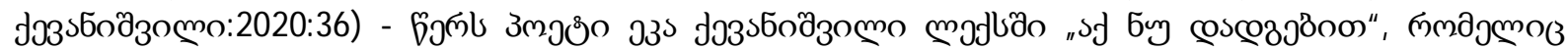

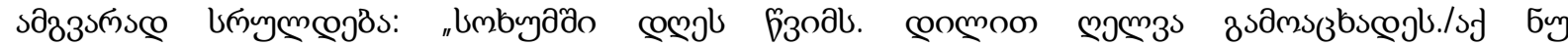

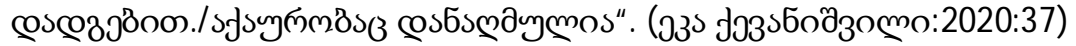

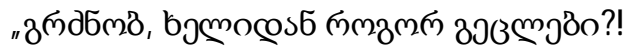

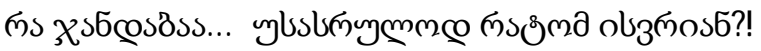

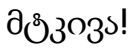

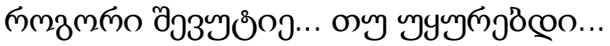

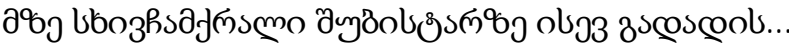

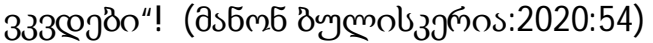

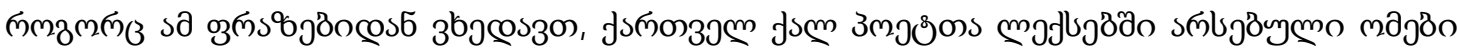

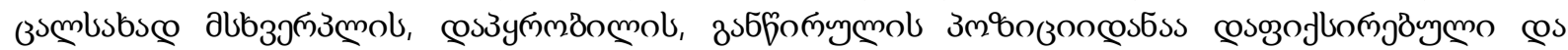

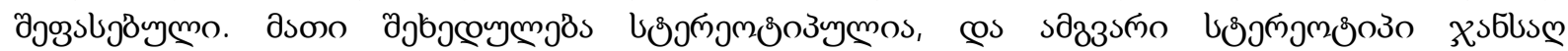




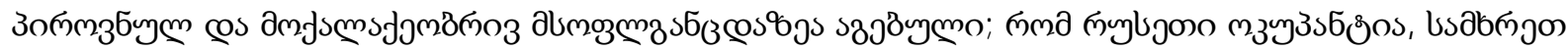

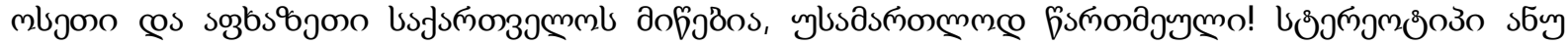

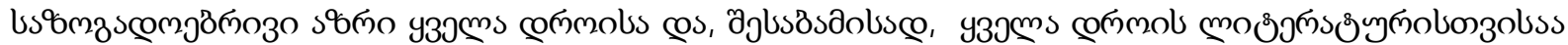

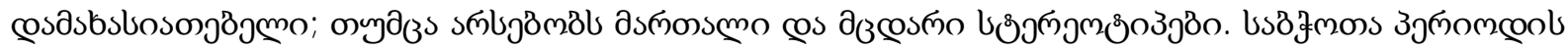

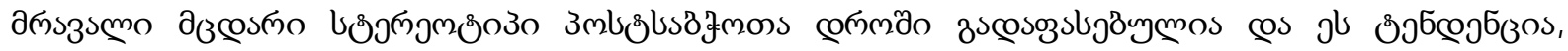

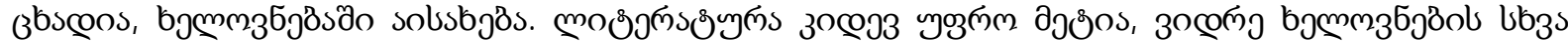

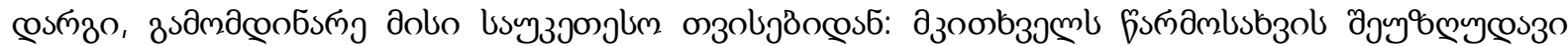

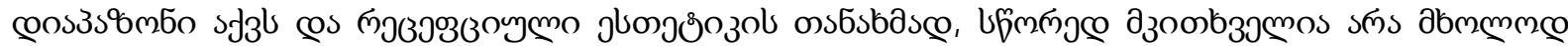

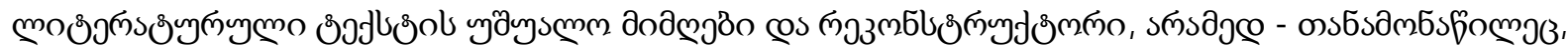

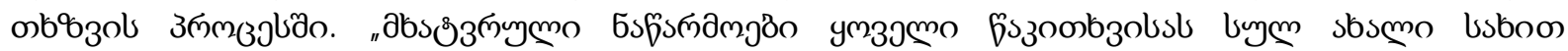

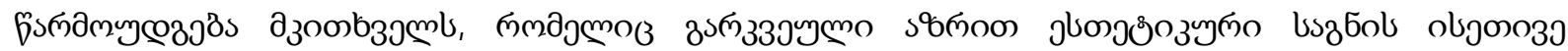

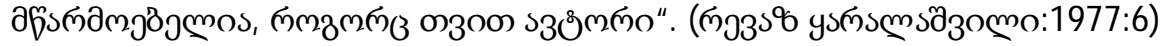

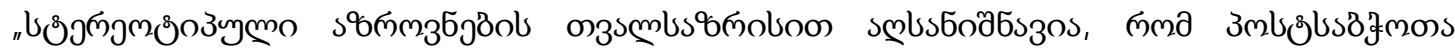

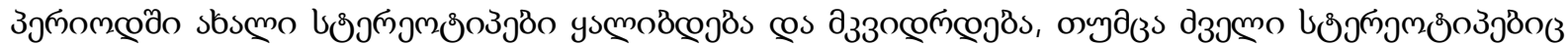

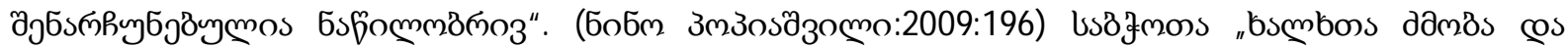

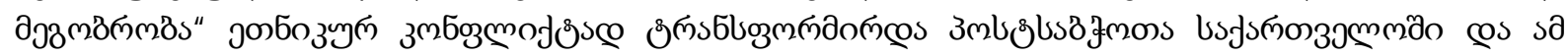

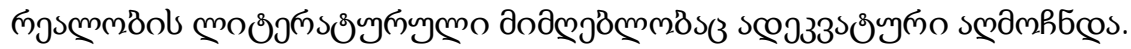

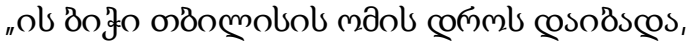

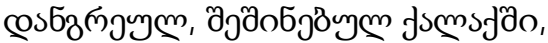

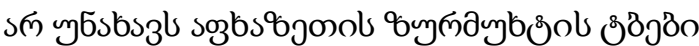

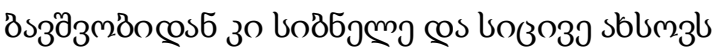

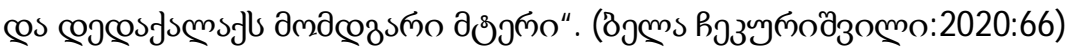

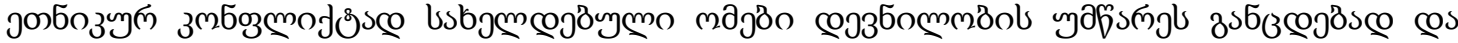

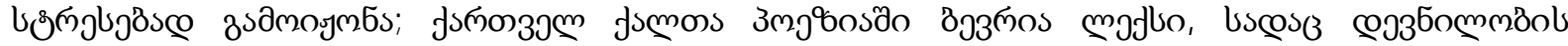

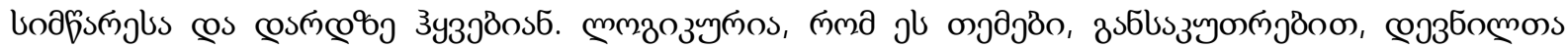
зmg bosðoo solsbs.

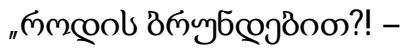 \\ so, дsল́sconymo zootbुs, \\ asmsconymsco

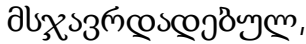 \\ "@ग3

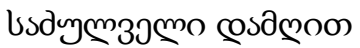

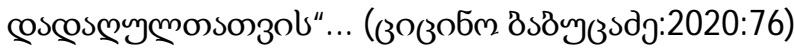

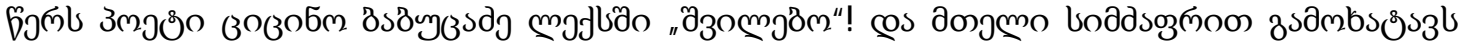

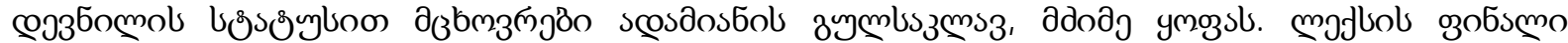

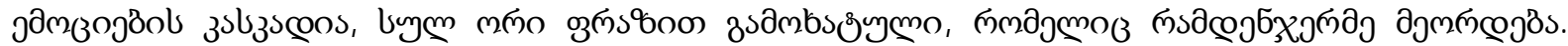

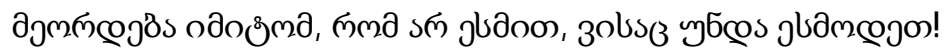

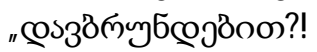

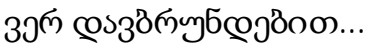

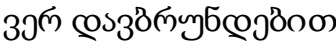

39

smslumegl fisamzlummzsmon!

snsbmegl fsamzluymzsmon!

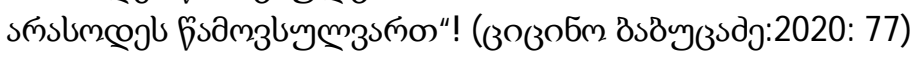

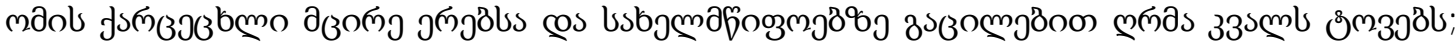

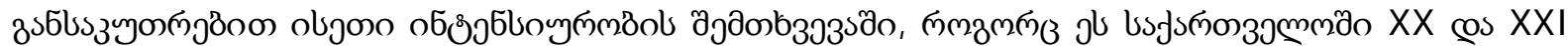

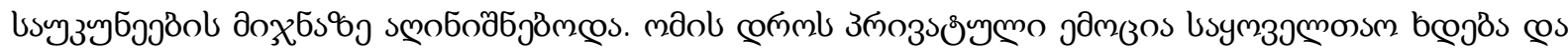

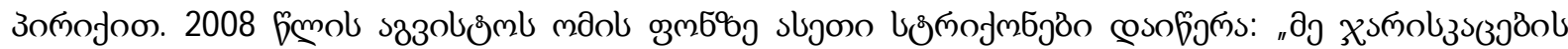

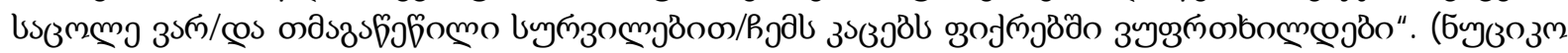

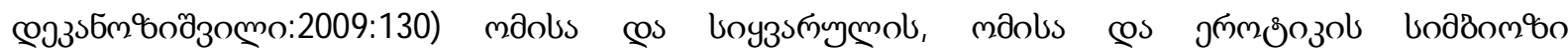




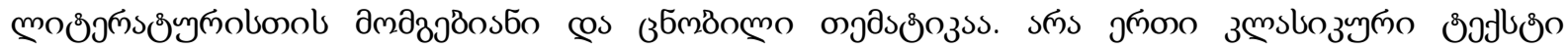

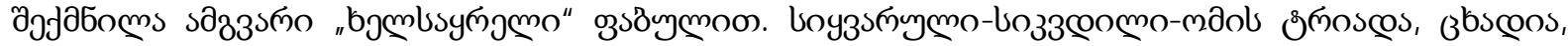

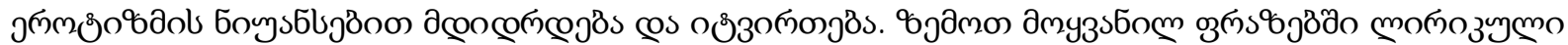

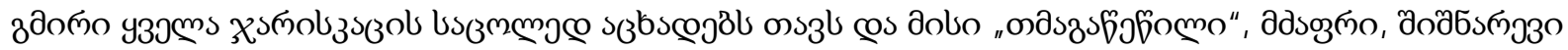

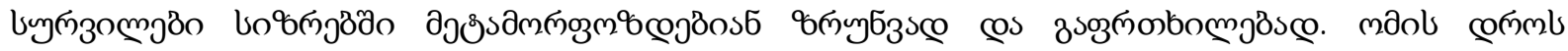

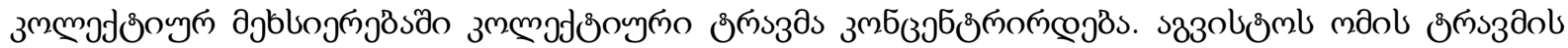

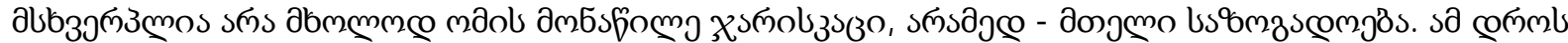

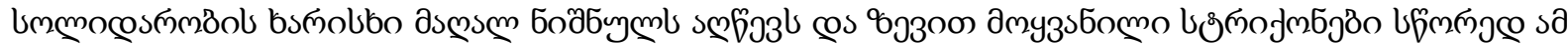

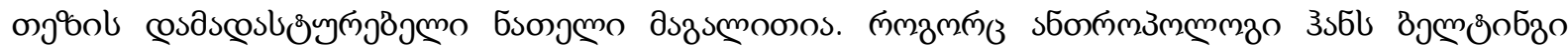

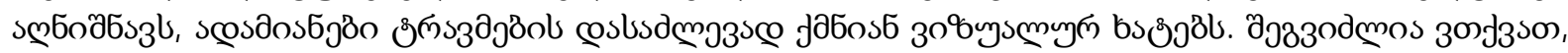

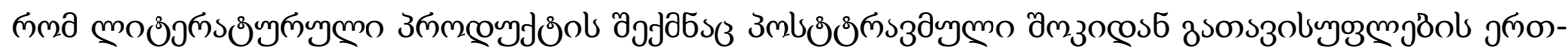

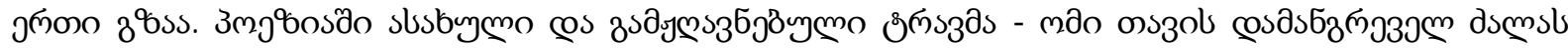

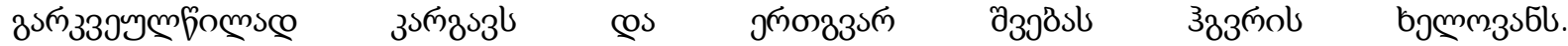

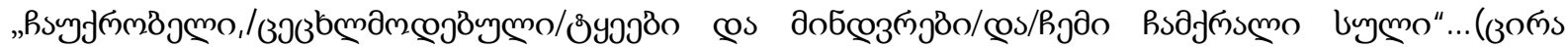

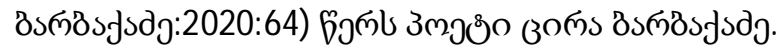

\section{"oyyghj...}

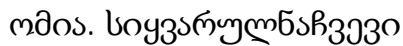

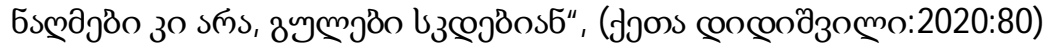

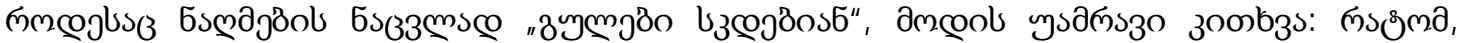

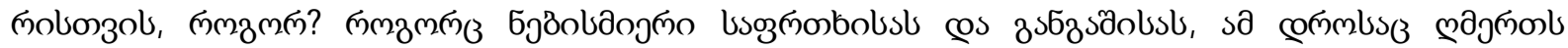

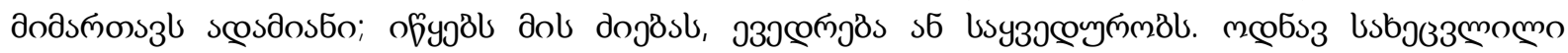

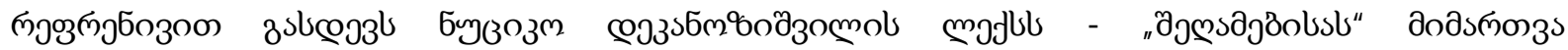

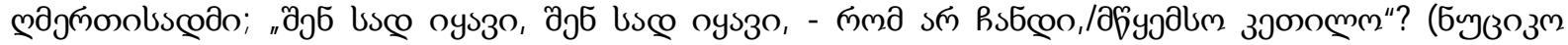

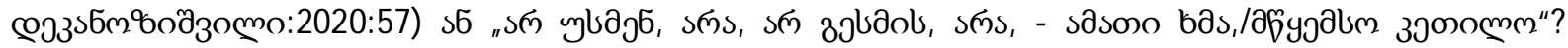

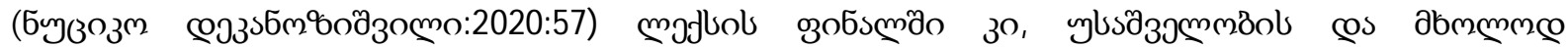

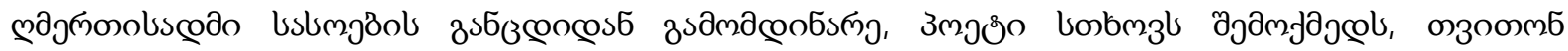

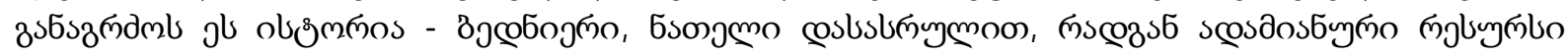
samfiymmymos:

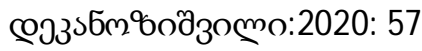

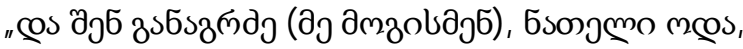

абудаим зуолмm!

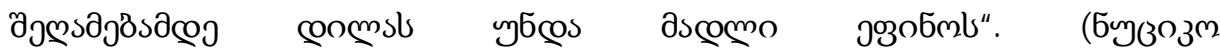

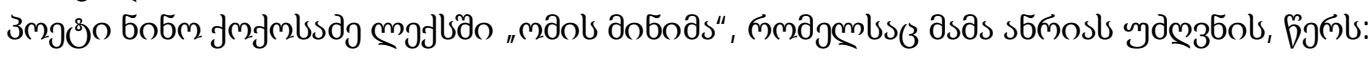

"- 3olos dofs?

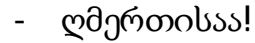

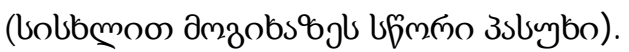

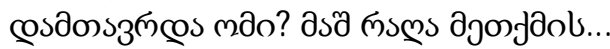

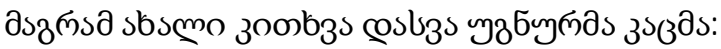

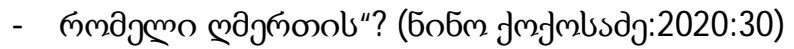

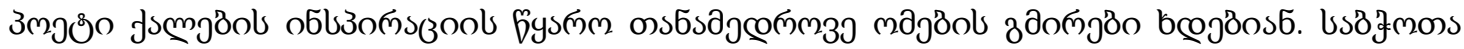

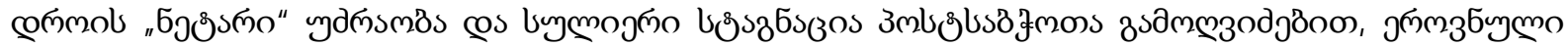

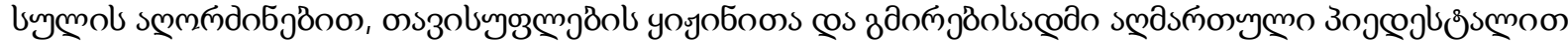

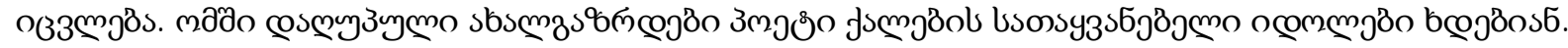

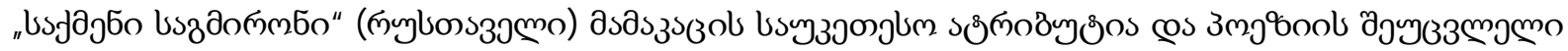

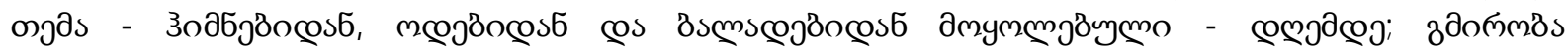

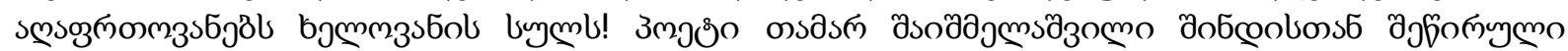

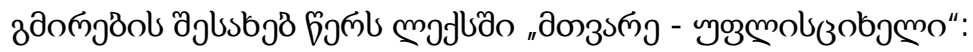

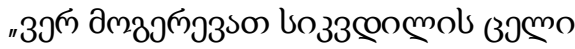




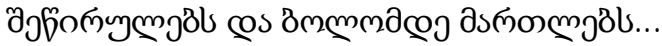

cos ylsbjmm yogmolsobjmo -

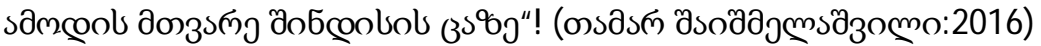

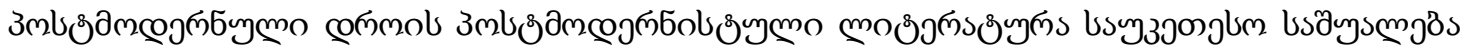

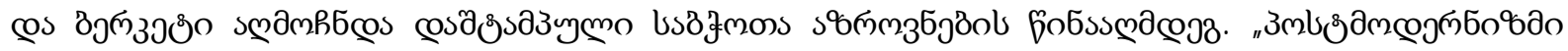

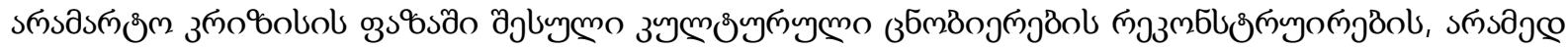

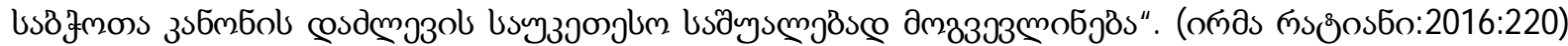

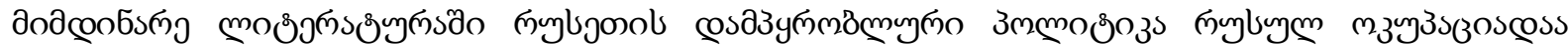

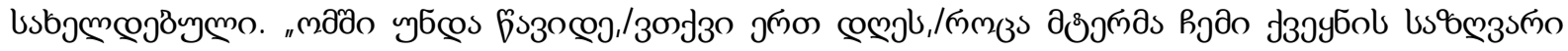

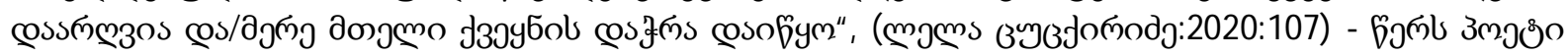

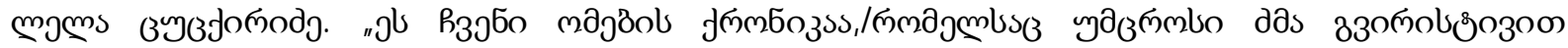

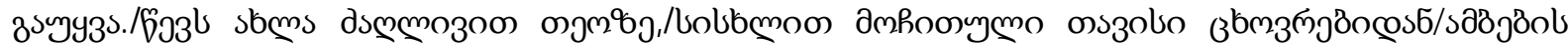

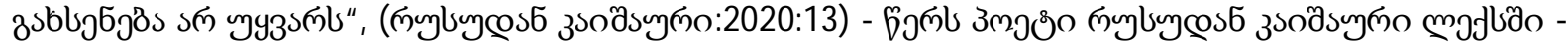

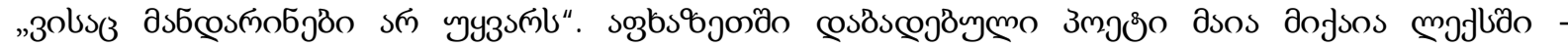

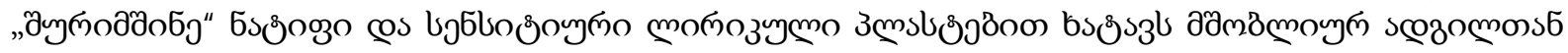

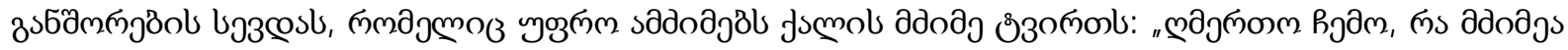

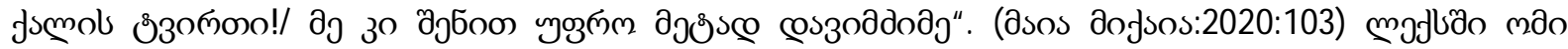

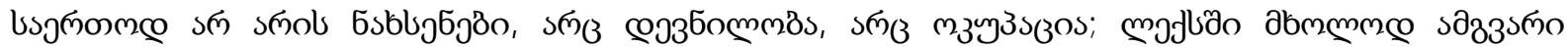

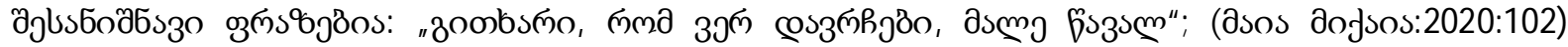

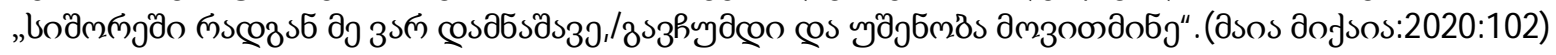

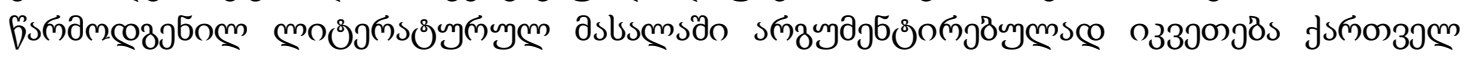

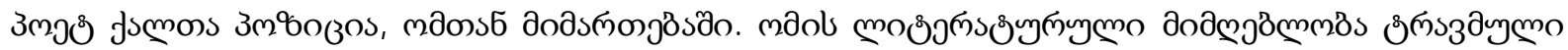

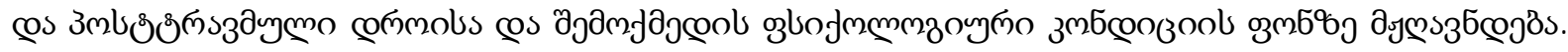

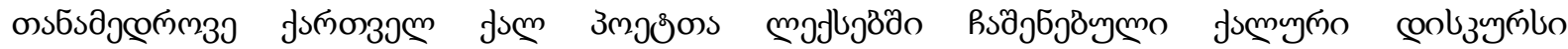

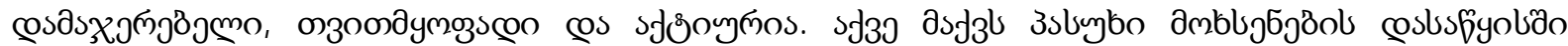

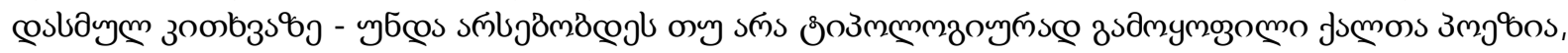

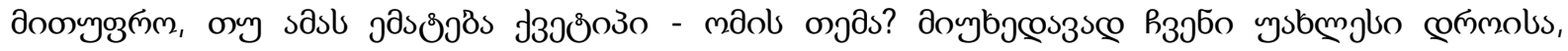

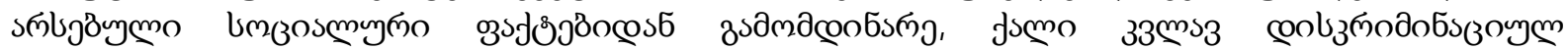

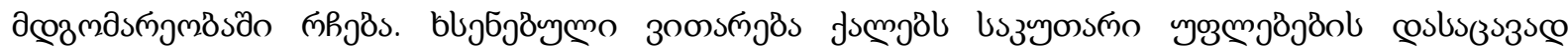

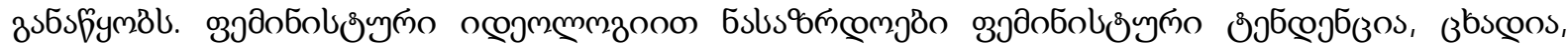

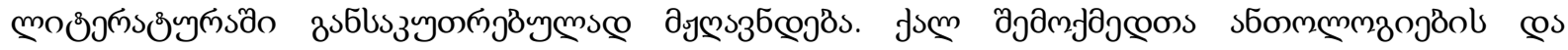

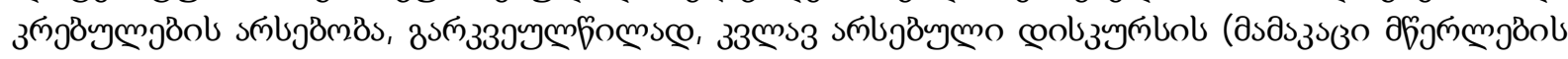
зпомпо о

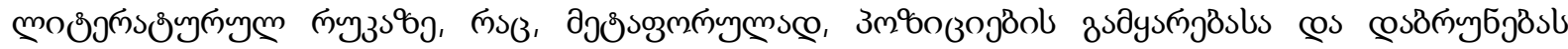

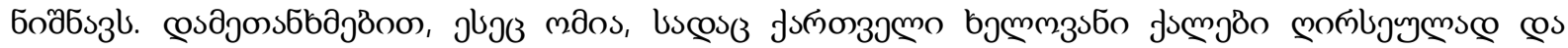

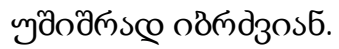

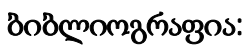

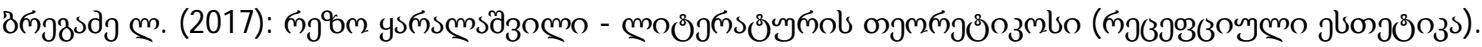

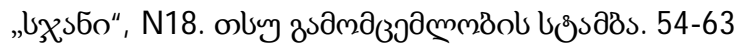

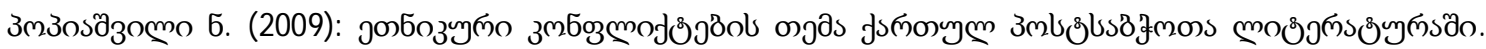

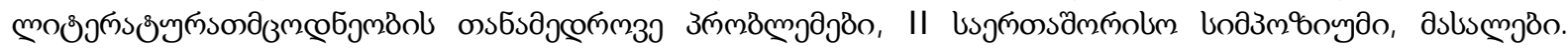

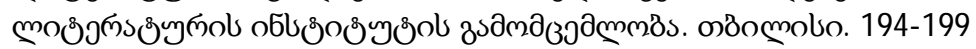

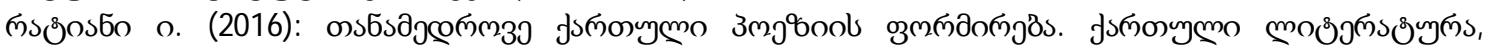

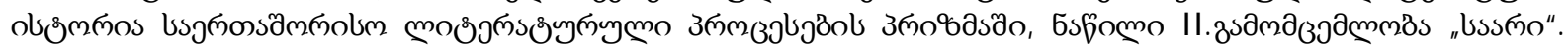
कठ̀nmolo. 211-220

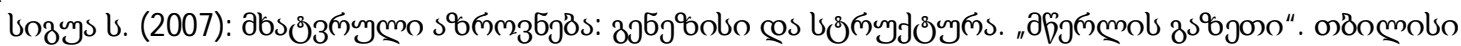
ys пேs ms 
lss6smo

oв̈omolo

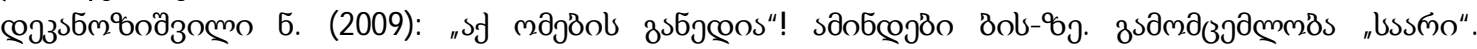

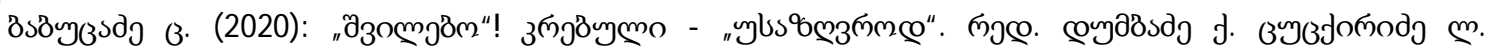

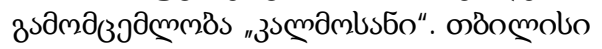

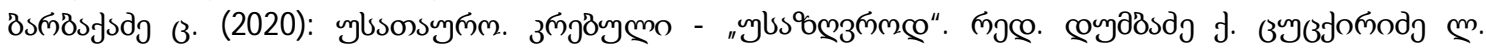

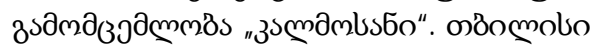

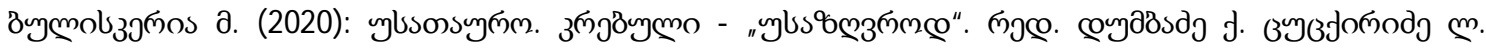

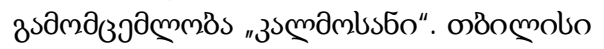

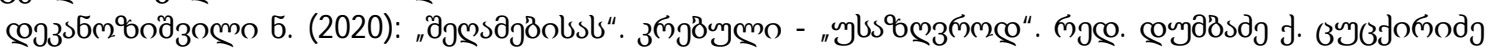

м. зsамда

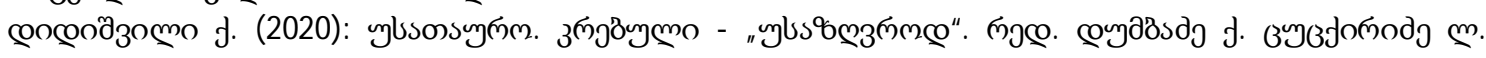

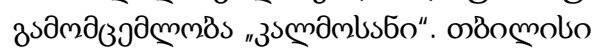

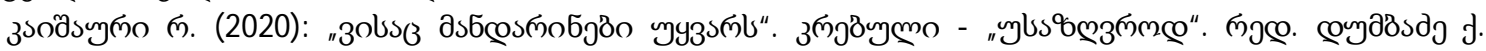

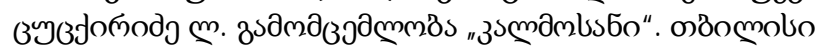

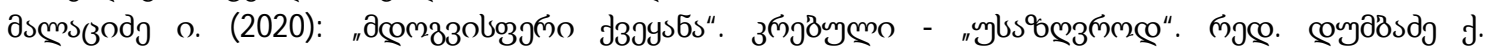

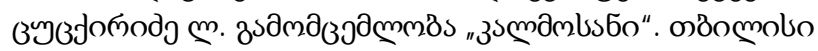

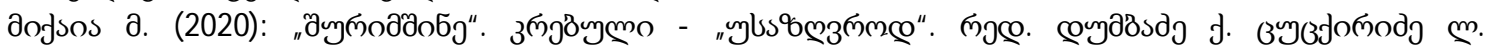

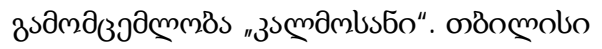

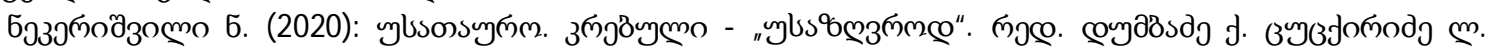

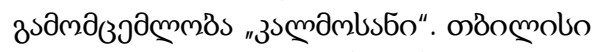

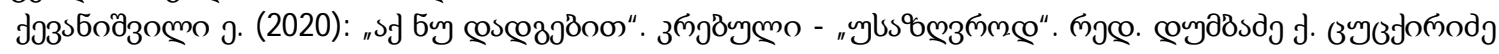
м. зsдмд

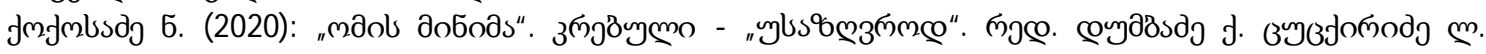

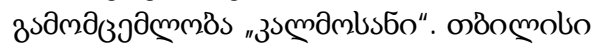

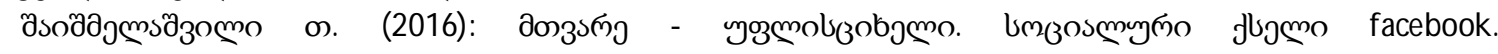
https://www.facebook.com/photo/?fbid=1151223268270024\& set=a.769888363070185

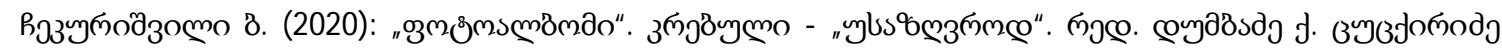

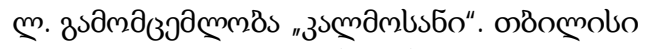

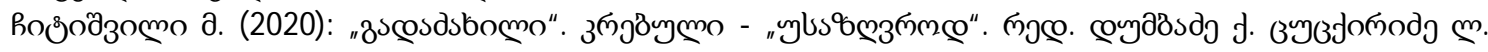

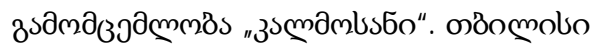

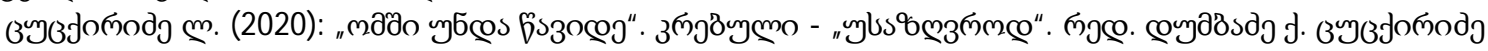

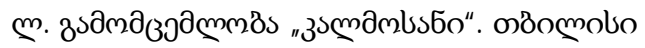

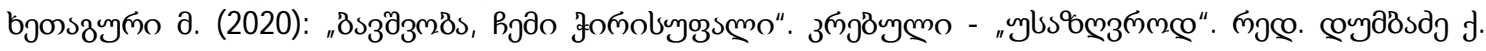

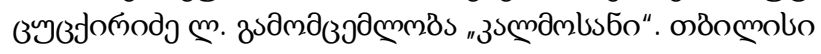

\title{
Literary Reception of War in the Contemporary Georgian Women's Poetry
}

\author{
Gogiashvili Nino \\ Iakob Gogebashvili State University, Telavi
}

\begin{abstract}
War has been reflected in national cultures and literature of every country, as it is related to sharp and turbulent emotions. The fear of death, tension, heroic pathos and suffering, following every war, gives it esthetic value and certain romantic touch too. Georgian-Ossetian conflict, war in Abkhazia, civil war and Georgian-Russian war have clearly been reflected in the literature created at the turn of the XX-XXI centuries, both in prose and poetry. In the presented report I will discuss Georgian women's poetry, in which war is a literary reception and poems are the space for the reflection of emotions caused by war.

In 2020, were published 2 volumes of the Almanac - Without Limits - which include texts by Georgian woman poets and writers, dedicated to war. Accordingly, the main literary material when
\end{abstract}


preparing the report was the first, poetry volume of the aforementioned Almanac.

The thesis does not consider discussion of war reception in general, in contemporary poetry, but only in the works by contemporary woman poets. There are radically different opinions on whether or not anthologies must differ according to gender and that art and its creator - artist - do not have an art-gender. It is true that art is universal and stands above any ethnic, race, religious, gender or age affiliations; however, we cannot ignore the fact that all these criteria are revealed themselves in literary works. Therefore, women's poetry is specific and woman is always seen in its invisible nuances. In view of the research, it appeared to be very interesting and essential, how the war topic has been accepted and processed by contemporary women's poetry.

Contexts of Russian occupation, Georgian-Abkhazian and Georgian-Ossetian ethnic conflicts, civil wars, formed as the new, post-soviet stereotypes, have clearly been reflected in Georgian women's poetry; while postmodernism has appeared to be the favorable space for ignoring the Soviet clichés.

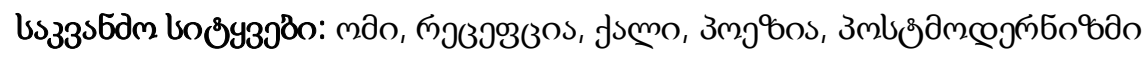

Keywords: War, Reception, Woman, Poetry, Postmodernism 